By the interior is meant all points of the plane which are not on the curve but which are separated from the point $\rho=0$ by the curve. By the exterior is meant all other points of the plane not on the curve nor in the interior of the curve.

THEOREM 3. If, with the hypothesis of Theorem 2 , the $2 q$ radial lines through $\rho=0$ and the roots of $f^{\prime}(z)$ and midway between the roots are drawn so that the plane is divided into $2 q$ sectors, numbered from 1 to $2 q$, then either all the roots of $f(z)$ must lie on the radial lines or there must be roots in an odd as well as in an even numbered sector.

Covina, CALIF.

\title{
ON THE REPRESENTATIONS, $N_{7}\left(m^{2}\right)^{1}$
}

\section{D. OLDS}

1. Introduction. Write $N_{r}(n)$ for the number of representations of the positive integer $n$ as the sum of $r$ squares, and write $N_{r}(n, k)$ for the number of representations of $n$ as the sum of $r$ squares in which the first $k$ squares in each representation are odd with positive roots, while the remaining $r-k$ squares are even with roots positive, negative, or zero. In a previous paper the author $[5]^{2}$ gave an arithmetical derivation of the formula for $N_{3}\left(n^{2}\right)$. The method used to prove this result was based upon that employed by Hurwitz [2] in his discussion of the analogous formula for $N_{5}\left(n^{2}\right)$.

In 1930, G. Pall [6] gave an analytical derivation of the formula for $N_{7}\left(c n^{2}\right), c$ an integer. His formula shows, in particular, that if $m=p_{1}^{\alpha_{1}} p_{2}^{\alpha_{2}} \cdots p_{s}^{\alpha_{s}}$, where $p_{1}, p_{2}, \cdots, p_{s}$ are distinct odd primes, then

$$
N_{7}\left(m^{2}\right)=14 \prod_{\nu=1}^{s}\left[p_{\nu}, \alpha_{\nu}\right]
$$

where

$$
\left[p_{\nu}, \alpha_{\nu}\right]=\sigma_{5}\left(p_{\nu}^{\alpha_{\nu}}\right)-(-1)^{\left(p_{\nu}-1\right) / 2}{ }_{p_{\nu}}^{2} \sigma_{5}\left(p_{\nu}^{\alpha_{\nu}-1}\right) .
$$

We define the arithmetical function $\sigma_{k}(n)$, which occurs here, and the function $\rho_{k}(n)$, which occurs later, by the sums

${ }_{1}^{1}$ This is the second part of a paper presented to the Society, April 6,1940, under the title On the number of representations of the square of an integer as the sum of an odd number of squares. The author wishes to thank Professor J. V. Uspensky for help in preparing this paper.

${ }^{2}$ The numbers in brackets refer to the bibliography. 
194I]

$$
\sigma_{k}(n)=\sum_{d \mid n} d^{k}, \quad \rho_{k}(n)=\sum_{n=d \delta}(-1)^{(\delta-1) / 2} d^{k}, \quad \delta \text { odd, }
$$

where in the first sum $d$ ranges over all divisors of $n: 1 \leqq d \leqq n$, and in the second $d, \delta$ range over all integral solutions of the equation $n=d \delta$, where $\delta$ is odd. It is the purpose of this paper to show how formula (1) may be derived arithmetically. We shall use the method of Hurwitz [2].

2. Preliminary formulas. In the proof of (1) which follows, we use certain well known results concerning $N_{r}(n)$, when $r$ is even. These formulas, which are not difficult to prove arithmetically, ${ }^{3}$ are

$$
N_{6}(m)=12 \rho_{2}(m), \quad m \equiv 1(\bmod 4) ;
$$

(3) $N_{6}(2 m, 2)=\rho_{2}(m), N_{6}\left(2^{\alpha+2} m, 4\right)=2^{2 \alpha} \rho_{2}(m)=\chi\left(2^{\alpha} m\right), \alpha \geqq 0, m$ odd;

$$
N_{12}(4 m, 4)+16 N_{12}(4 m, 8)=\sigma_{5}(m), \quad m \text { odd. }
$$

3. Three lemmas. The first lemma we need was proved by the author in [5].

Lemma 1. Let $f(n)$ be an arbitrary arithmetical function, not equal to zero for all $n$, and such that $f\left(n n^{\prime}\right)=f(n) f\left(n^{\prime}\right)$ for any two integers $n$ and $n^{\prime}$. If $F(n)=\sum f(d)$, where $d$ ranges over all divisors of $n$, then

$$
F\left(n n^{\prime}\right)=\sum_{(a)} \mu(d) f(d) F(n / d) F\left(n^{\prime} / d\right),
$$

where (a) indicates that we take $d=1,2,3, \cdots$, with the convention that $F(x)=0$ if $x$ is not an integer. Here $f(1)=F(1)=1$ and $\mu(d)$ denotes the Möbius function.

Now let $P=N_{7}\left(m^{2}\right), m$ an odd integer, and let $P_{1}$ denote the number of solutions of the equation

$$
m^{2}=x^{2}+y^{2}+z^{2}+t^{2}+u^{2}+v^{2}+4 w^{2},
$$

and $P_{2}$ the number of solutions of the equation

$$
m^{2}=\bar{x}^{2}+\bar{y}^{2}+\bar{z}^{2}+\bar{t}^{2}+\bar{u}^{2}+4 v^{2}+4 w^{2},
$$

where the barred squares have odd roots and occupy the first five places in each representation. Then it may be proved without difficulty that

$$
P=\frac{7}{6}\left(P_{1}+12 P_{2}\right),
$$

\footnotetext{
${ }^{3}$ Formula (2) may be found, for example, in J. Liouville [3], formulas (3) and (4) in Liouville [4]. For the method used to prove such formulas arithmetically, see T. Pepin [7] or P. Bachmann [1].
} 
and thus our problem is reduced to the determination of convenient expressions for $P_{1}$ and $P_{2}$.

We first discuss $P_{1}$. To this end, notice that

$$
P_{1}=\sum_{(b)} N_{6}\left(m^{2}-4 w^{2}\right)
$$

where $(b)$ indicates that $w=0, \pm 1, \pm 2, \cdots ; 4 w^{2}<m^{2}$. On the other hand, (2) shows us that

$$
N_{6}\left(m^{2}-4 w^{2}\right)=12 \rho_{2}\left(m^{2}-4 w^{2}\right),
$$

so that

$$
\begin{aligned}
P_{1} & =12 \sum_{(b)} \rho_{2}\left(m^{2}-4 w^{2}\right)=12 \sum_{(b)} \rho_{2}((m-2 w)(m+2 w)) \\
& =12 \sum_{(c)} \rho_{2}\left(m^{\prime} m^{\prime \prime}\right),
\end{aligned}
$$

where the last sum ranges over all positive odd integers $m^{\prime}, m^{\prime \prime}$ satisfying the equation $(c): 2 m=m^{\prime}+m^{\prime \prime}$.

To evaluate $\rho_{2}\left(m^{\prime} m^{\prime \prime}\right)$ use Lemma 1 . Define

$$
\begin{aligned}
& f(n)=0, \text { whenever } n \text { is even, } \\
& f(n)=(-1)^{(n-1) / 2} n^{2}, \quad \text { whenever } n \text { is odd; }
\end{aligned}
$$

then $f\left(n n^{\prime}\right)=f(n) f\left(n^{\prime}\right)$ for any two integers $n, n^{\prime}$ as required, and for odd $n(=m)$,

$$
\begin{array}{rlr}
F(m) & =\sum_{d \mid m} f(d)=\sum_{d \mid m}(-1)^{(d-1) / 2} d^{2}=(-1)^{(m-1) / 2} \sum_{m=d \delta}(-1)^{(\delta-1) / 2} d^{2} \\
& =(-1)^{(m-1) / 2} \rho_{2}(m), & \delta \text { odd. }
\end{array}
$$

Applying (5) we obtain

$$
\rho_{2}\left(m^{\prime} m^{\prime \prime}\right)=\sum_{(a)} \mu(d)(-1)^{(d-1) / 2} d^{2} \rho_{2}\left(m^{\prime} / d\right) \rho_{2}\left(m^{\prime \prime} / d\right),
$$

provided we adopt the convention that $\rho_{2}(x)=0$ if $x$ is not an integer. Hence

$$
\begin{aligned}
P_{1} & =12 \sum_{(c)} \rho_{2}\left(m^{\prime} m^{\prime \prime}\right)=12 \sum_{(c)} \sum_{(a)} \mu(d)(-1)^{(d-1) / 2} d^{2} \rho_{2}\left(m^{\prime} / d\right) \rho^{2}\left(m^{\prime \prime} / d\right) \\
& =12 \sum_{d=1,3,5, \cdots} \mu(d)(-1)^{(d-1) / 2} d^{2} \sum_{(c)} \rho_{2}\left(m^{\prime} / d\right) \rho_{2}\left(m^{\prime \prime} / d\right) .
\end{aligned}
$$

Finally, with the aid of (3), we find

$$
\sum_{(c)} \rho_{2}\left(\frac{m^{\prime}}{d}\right) \rho_{2}\left(\frac{m^{\prime \prime}}{d}\right)=\sum_{(c)} N_{6}\left(\frac{2 m^{\prime}}{d}, 2\right) N_{6}\left(\frac{2 m^{\prime \prime}}{d}, 2\right)=N_{12}\left(\frac{4 m}{d}, 4\right) .
$$


Thus we have established

Lemma 2. $P_{1}=12 \sum_{d \mid m} \mu(d)(-1)^{(d-1) / 2} d^{2} N_{12}(4 m / d, 4)$.

We evaluate $P_{2}$ in a similar manner. Since

$$
P_{2}=2^{4} \sum_{(d)} N_{6}\left(m^{2}-x^{2}, 4\right),
$$

where the extent of the summation is indicated by $(d): x= \pm 1$, $\pm 3, \cdots ; x^{2}<m^{2}$; we find, using (3), that

$$
\begin{aligned}
P_{2} & =16 \sum_{(d)} \chi\left(\frac{m^{2}-x^{2}}{4}\right)=16 \sum_{(d)} \chi\left(\frac{m+x}{2} \cdot \frac{m-x}{2}\right) \\
& =16 \sum_{(e)} \chi\left(n^{\prime} n^{\prime \prime}\right),
\end{aligned}
$$

where $n^{\prime}, n^{\prime \prime}$ are any two positive integers satisfying the equation $(e)$ : $m=n^{\prime}+n^{\prime \prime}$. Setting

$$
n^{\prime}=2^{\alpha} m^{\prime}, \quad n^{\prime \prime}=2^{\beta} m^{\prime \prime},
$$

where $m^{\prime}, m^{\prime \prime}$ are odd, and using (6), we deduce that

$$
\begin{aligned}
\chi\left(n^{\prime} n^{\prime \prime}\right) & =2^{2(\alpha+\beta)} \rho_{2}\left(m^{\prime} m^{\prime \prime}\right) \\
& =\sum_{(a)} \mu(d)(-1)^{(d-1) / 2} d^{2} 2^{2 \alpha} \rho_{2}\left(\frac{m^{\prime}}{d}\right) 2^{2 \beta} \rho_{2}\left(\frac{m^{\prime \prime}}{d}\right) \\
& =\sum_{(a)} \mu(d)(-1)^{(d-1) / 2} d^{2} \chi\left(\frac{n^{\prime}}{d}\right) \chi\left(\frac{n^{\prime \prime}}{d}\right) .
\end{aligned}
$$

As a result

$$
P_{2}=16 \sum_{(e)} \chi\left(n^{\prime} n^{\prime \prime}\right)=16 \sum_{d=1,3,5, \cdots} \mu(d)(-1)^{(d-1) / 2} d^{2} \sum_{(e)} \chi\left(\frac{n^{\prime}}{d}\right) \chi\left(\frac{n^{\prime \prime}}{d}\right) .
$$

Now by (3)

$$
\sum_{(e)} \chi\left(\frac{n^{\prime}}{d}\right) \times\left(\frac{n^{\prime \prime}}{d}\right)=\sum_{(e)} N_{6}\left(\frac{4 n^{\prime}}{d}, 4\right) N_{6}\left(\frac{4 n^{\prime \prime}}{d}, 4\right)=N_{12}\left(\frac{4 m}{d}, 8\right) .
$$

We have proved

Lemma 3. $P_{2}=16 \sum_{d \mid m} \mu(d)(-1)^{(d-1) / 2} d^{2} N_{12}(4 m / d, 8)$.

4. Derivation of the formula for $N_{7}\left(m^{2}\right)$. Since we know already $P=\frac{7}{6}\left(P_{1}+12 P_{2}\right)$, we find, using Lemma 2 , Lemma 3 , and finally (4), that 


$$
\begin{aligned}
P= & \frac{7}{6}\left[12 \sum_{d \mid m} \mu(d)(-1)^{(d-1) / 2} d^{2} N_{12}\left(\frac{4 m}{d}, 4\right)\right. \\
& \left.+12 \cdot 16 \sum_{d \mid m} \mu(d)(-1)^{(d-1) / 2} d^{2} N_{12}\left(\frac{4 m}{d}, 8\right)\right] \\
= & 14 \sum_{d \mid m} \mu(d)(-1)^{(d-1) / 2} d^{2}\left[N_{12}\left(\frac{4 m}{d}, 4\right)+16 N_{12}\left(\frac{4 m}{d}, 8\right)\right] \\
= & 14 \sum_{d \mid m} \mu(d)(-1)^{(d-1) / 2} d^{2} \sigma_{5}\left(\frac{m}{d}\right) \\
= & 14 \prod_{\nu=1}^{s}\left[p_{\nu}, \alpha_{\nu}\right],
\end{aligned}
$$

where

$$
\left[p_{\nu}, \alpha_{\nu}\right]=\sigma_{5}\left(p_{\nu}^{\alpha_{\nu}}\right)-(-1)^{\left(p_{\nu}-1\right) / 2} p_{\nu}^{2} \sigma_{5}\left(p_{\nu}^{\alpha_{\nu}-1}\right) .
$$

This is the result we set out to prove.

\section{BIBLIOGRAPHY}

1. P. Bachmann, Niedere Zahlentheorie, Part 2, Leipzig, 1910, pp. 365-433.

2. A. Hurwitz, Comptes Rendus de l'Académie des Sciences, Paris, vol. 98 (1884), pp. 504-507. (Extract from a letter addressed to Hermite.)

3. J. Liouville, Sur un certain genre de décompositions d'un entier en sommes de carrés, Journal de Mathématiques, (2), vol. 6 (1861), p. 236.

4. J. Liouville, Les fonctions $N(n, p, q)$ et d'autres fonctions qui s'y rattachent, ibid., p. 371.

5. C. D. Olds, On the representations, $N_{3}\left(n^{2}\right)$, this Bulletin, vol. 47 (1941), pp. 499-503.

6. G. Pall, On the number of representations of a square, or a constant times a square, as the sum of an odd number of squares, Journal of the London Mathematical Society, vol. 5 (1930), pp. 102-105.

7. T. Pepin, Journal de Mathématiques Pures et Appliquées, (4), vol. 4 (1888), pp. 83-127.

Purdue University 